\title{
Improvement in Survival Outcomes of Acute \\ Lymphoblastic Leukemia Patients Treated With \\ Berlin-Frankfurt-Munster 76/79 Protocol
}

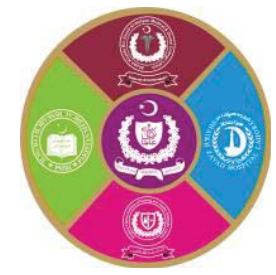

${ }^{1}$ Khansa Saleem, ${ }^{1}$ Muhammad Ilyas, ${ }^{1}$ Saad Razzaq, ${ }^{1}$ Fahad Maqbool, ${ }^{1}$ Qaiser Abbas,

${ }^{2}$ Aneem Abdullah, ${ }^{3}$ Abu Bakar Shahid

${ }^{1}$ Department of Computer Sciences\& Information Technology, University of Sargodha, Sargodha

${ }^{2}$ Department of Nephrology, Shaikh Zayed Medical Complex, Lahore

${ }^{3}$ INMOL Cancer Hospital, Lahore

\begin{abstract}
trat
ABSTRACT

Introduction: Acute lymphoblastic leukemia (ALL) is a malignancy of the white blood cells and lymphoid lines of hematopoietic cells in the blood and bone marrow while lymphomas mostly involve the lymph nodes. This study showed the response rate of ALL treated with BFM (Berlin-Frankfurt-Munster) 76/79 protocol. Aims \& Objectives: To study the effects of WBC (White Blood Cells), age, and treatment protocols on the survival of ALL patients to BFM 76/79 protocol and other chemotherapy protocol. Place and duration of study: 6 months retrospective study on 2015-2017 indoor patient data from INMOL Hospital Lahore, Pakistan. Material \& Methods: Data of 129 patients was assessed for eligibility, and 84 patients of ALL selected. Clinical information of patients regarding Gender, Age, WBC, Fever, Hepatosplenomegaly, Lymphadenopathy, Bleeding, Bone Pain, Immunophenotype, Relapse, Death, and survival rates were recorded. Patients were grouped according to Age, WBC, Gender, and Immunophenotype. Patients were treated with BFM 76/79 Protocols and the remaining patients were treated with other protocols (CALGB, FLAG-TDA, Hyper-CVAD, EURO, COG, T-Cell ALL protocol, and ALL protocol 9111). Results: Out of 84 patients, $55(65.5 \%)$ patients were treated with Berlin-Frankfurt-Munster 76/79 protocol and the remaining $20(23.81 \%)$ patients with other protocols. After the end of induction $64(73.8 \%)$ patients achieved complete remission and 5 (5.95\%) patients did not achieve Remission. Conclusion: Response rate of ALL (Acute Lymphoblastic Leukemia) patients treated with BFM 76/79 protocol was better than other protocols and possibly when risk adapted for male gender, $\mathrm{WBC}>50 \times 10^{9} / \mathrm{L}$ and age $>30$ years.
\end{abstract}

Keywords: Acute Lymphoblastic Leukemia (ALL), BFM protocols, Acute Myeloid Leukemia (AML), NonHodgkin Lymphoma (NHL)

\section{INTRODUCTION}

\begin{abstract}
A cute Lymphoblastic Leukemia is a cancerous disease of the bone marrow where immature leucocytes and lymphoid precursors proliferate and replace the normal hematopoietic cells and immune system B and T cells. Whereas lymphatic tissue and lymph nodes malignancy is preponderant in lymphomas. Research has revealed that most of the deaths and poor outcomes of leukemia and lymphoma patients were due to many reasons i.e. lack of awareness about the disease, lack of early treatment of disease, the toxic effect of protocol or chemotherapy treatment, and poor socioeconomic condition of patients. Studies have shown that major risk factors involved in poor outcomes of Leukemia patients were, WBC $>50 \times 10^{9} / \mathrm{L}$, Male Gender,
\end{abstract}

Age, and poor socioeconomic condition..$^{1,2}$ Heredity may also play a role as a leukemic gene ETV6RUNX1 could arise in one of the twins in the womb and twins can develop ALL. ${ }^{3}$ Following chemotherapy the rate of Induction Remission varies with different ALL protocols. In case of the BFM protocol it was found to be $86.6 \%$ which is greater than as compared to the outcome of $85 \%$ of UKALL-X protocol. ${ }^{2} 86 \%$ patients, when treated with BFM protocol, showed complete remission, in comparison to $62 \%$ overall survival for T-Cell ALL patients and 38\% B-Cell ALL patients using CALGB. ${ }^{4}$ Risk-adapted therapy for Pre B-Cell and T-Cell ALL Children showed $86 \%$ of 5-year Eventfree survival rates and low relapse rate. $^{5}$ Mitoxantrone is an effective drug for relapse patients of ALL then "idarubicin". ${ }^{6}$ Fever, weakness were considered the commonest symptoms for 
Leukemia patients. $^{7}$ Toxicity is a major risk for long-term event-free-survival outcomes, so a balance in therapeutic treatment is required. ${ }^{8}$ It is suggested that Risk adapted therapies are beneficial for good outcomes of patients in ALL. ${ }^{5,9}$ Classification of Acute Leukemia according to FAB classification and World Health Organization was described so that the treatment should be given according to the diagnosis of a particular subtype. ${ }^{10}$ Proper treatment should be given to ALL patients to attain improvement in survival rates and to decrease the rate of deaths due to infectious diseases. ${ }^{11}$ High Relapse rate is a major problem in previous studies. In the present study, the relapse rate was controlled by risk adapted therapy.

\section{MATERIAL AND METHODS}

A 6 months retrospective study was conducted on 2015-2017 indoor patient data from INMOL Hospital Lahore, Pakistan. Data of 129 patients aged from 2-54 years (83.0\% patients between age 11 to 30 years) were assessed for eligibility on the basis ALL $(n=84)$, AML $(n=9)$ and NHL $(n=35)$ patients. 84 patients with ALL were selected for this study. Patients enrollment, allocation, follow up and analysis are detailed in the Patients Consort E Flow (Fig-1).

Complete information of patients containing their physical examination, biological and clinical features as well as the family history was recorded for analysis. clinical information of patients regarding Gender, Age, WBC, Fever, Hepatosplenomegaly, Lymphadenopathy, Bleeding, Bone Pain, Immunophenotype, Relapse, Death, and survival rates were recorded.

Patients were grouped according to the Age, Gender. WBC, and Immunophenotype and Treatment protocol. Patients were grouped for age (1-10 years, 11-20 years, 21-30 years, and greater than 30 years). Patients having $\mathrm{WBC}>50 \times 10^{9} / \mathrm{L}$, age $>30$ years, male gender, and T Cell ALL patients were considered in a High-Risk group, and patients having $\mathrm{WBC}<50 \times 10^{9} / \mathrm{L}$ were in a LowRisk Group. Immunophenotyping and FAB classification Leukemia subtypes were used for determining morphology and immunophenotype of patient's blood and Bone marrow samples. Grouping according to immunophenotyping e.g. Pre B-Cell and T-Cell patients were done separately. Due to meagre data on Philadelphia +ve chromosomes in these patients, it was excluded from analysis, and no risk assessment was done on it.

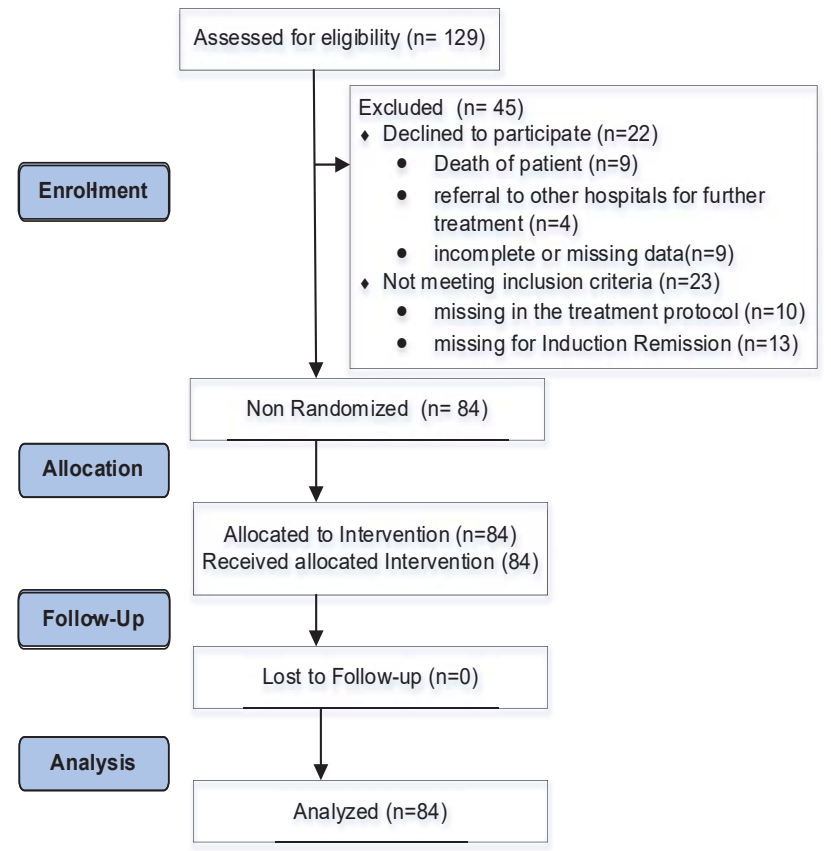

Fig-1: Patients Consort E chart

Treatment: Most of the patients were treated with BFM 76/79 Protocols and the remaining patients were treated with other protocols (CALGB, FLAGTDA, Hyper-CVAD, EURO, COG, T-Cell ALL protocol, and ALL protocol 9111).

\section{Statistical analysis:}

The data was analyzed statistically using MS Excel and SPSS 16.0 software Characteristics of patients were calculated and analysis of risk patients was done. Risk directed therapy protocols were used to analyze the survival rates of patients. Overall 4 year survival rate of leukemia patients was determined using MS Excel line charts after analyzing the deaths and relapse rates.

\section{RESULTS}

Out of 84 patients $65(77.3 \%)$ males and 19 (22.6\%) were females. The male to female ratio was 3.4:1. Clinical features of 84 patients with Acute Lymphoblastic Leukemia are shown in Table-1. The most common feature among patients was Fever; this was also proved by another study. ${ }^{7}$ The characteristics of patients are shown in Table-3.

\begin{tabular}{|l|c|c|}
\hline Features & YES & NO \\
\hline Fever & $70(83.3 \%)$ & $5(5.95 \%)$ \\
\hline Bleeding & $33(39.3 \%)$ & $10(11.9 \%)$ \\
\hline Hepatomegaly & $22(26.2 \%)$ & $26(31 \%)$ \\
\hline Splenomegaly & $36(42.9 \%)$ & $18(21.4 \%)$ \\
\hline Lymphadenopathy & $30(35.7 \%)$ & $5(5.95 \%)$ \\
\hline Bone Pain & $32(38.1 \%)$ & $6(7.14 \%)$ \\
\hline
\end{tabular}

Table-1: Clinical features of ALL Patients 


\begin{tabular}{|l|c|c|c|}
\hline $\begin{array}{c}\text { Patient's } \\
\text { record }\end{array}$ & $\begin{array}{c}\text { Complete } \\
\text { Remission }\end{array}$ & $\begin{array}{c}\text { Incomplete } \\
\text { Remission }\end{array}$ & $\begin{array}{c}\text { No } \\
\text { Record }\end{array}$ \\
\hline No. of patients & 64 & 5 & 13 \\
\hline$\%$ age & $73.8 \%$ & $5.95 \%$ & $15.5 \%$ \\
\hline
\end{tabular}

Table-2: Remission status of ALL patients

\begin{tabular}{|c|c|c|c|}
\hline Groups & $\begin{array}{c}\text { No. of } \\
\text { patients } \\
(n=84)\end{array}$ & \begin{tabular}{|c|} 
Percentage \\
of patients \\
$(\%)$
\end{tabular} & P-value \\
\hline $\begin{array}{l}\text { Gender } \\
\text { Male } \\
\text { Female } \\
\end{array}$ & $\begin{array}{l}65 \\
19 \\
\end{array}$ & $\begin{array}{l}77.4 \\
22.6\end{array}$ & \}$<0.05$ \\
\hline $\begin{array}{l}\text { WBC } \\
>50 \times 10^{9} / \mathrm{L} \\
<50 \times 10^{9} / \mathrm{L} \\
\end{array}$ & $\begin{array}{l}26 \\
58 \\
\end{array}$ & $\begin{array}{l}30.9 \\
69.0 \\
\end{array}$ & \}N.S \\
\hline $\begin{array}{l}\text { Age } \\
1-10 \\
11-20 \\
21-30 \\
>30 \\
\end{array}$ & $\begin{array}{c}9 \\
50 \\
20 \\
5\end{array}$ & $\begin{array}{l}10.7 \\
59.5 \\
23.8 \\
5.95\end{array}$ & $\{<0.05$ \\
\hline $\begin{array}{l}\text { Immunophenotype } \\
\text { Pre B-Cell ALL } \\
\text { B-Cell ALL } \\
\text { Pre T-Cell ALL } \\
\text { T-Cell ALL }\end{array}$ & $\begin{array}{c}49 \\
2 \\
10 \\
3\end{array}$ & $\begin{array}{l}58.3 \\
2.38 \\
11.9 \\
3.57\end{array}$ & N.S \\
\hline \begin{tabular}{|l|} 
FAB Classification \\
L1 \\
L2 \\
L3
\end{tabular} & $\begin{array}{c}2 \\
16 \\
2\end{array}$ & $\begin{array}{l}2.38 \\
19.0 \\
2.38 \\
\end{array}$ & \}N.S \\
\hline $\begin{array}{l}\text { Treatment Protocol } \\
\text { BFM 76/79 } \\
\text { CALGB } \\
\text { ALL Protocol } 9111 \\
\text { FLAG TDA } \\
\text { Hyper-CVAD } \\
\text { T-Cell Protocol } \\
\text { EURO Protocol } \\
\text { COG Protocol } \\
\end{array}$ & $\begin{array}{c}55 \\
7 \\
1 \\
1 \\
1 \\
4 \\
2 \\
4\end{array}$ & $\begin{array}{l}65.5 \\
8.33 \\
1.19 \\
1.19 \\
1.19 \\
4.76 \\
2.38 \\
4.76 \\
\end{array}$ & $\left\{\begin{array}{l}\}<0.05 \\
\} \\
\} \\
\xi \\
\xi \\
\xi\end{array}\right.$ \\
\hline $\begin{array}{l}\text { Risk Group } \\
\text { Low Risk Patients } \\
\text { High Risk Patients } \\
\end{array}$ & $\begin{array}{l}58 \\
26 \\
\end{array}$ & $\begin{array}{l}69.0 \\
30.9 \\
\end{array}$ & \}N.S \\
\hline Overall Survival & 75 & 89.9 & \\
\hline
\end{tabular}

Table-3: Characteristic of ALL Patients

\section{DISCUSSION}

Clinical features of ALL patients were taken after the $\mathrm{CBC}$ report before Induction. A complete history of the patient as well as their physical condition, blood, and bone marrow examination was recorded. $83.3 \%$ of patients have a fever, a common clinical feature found in most of the patients, $39.3 \%$ had bleeding, (26.2\%) hepatomegaly, (42.9\%) Splenomegaly, $(35.7 \%)$ lymphadenopathy and $(38.1 \%)$ bone pain (Table-1). In our study 26 $(30.9 \%)$ patients had $\mathrm{WBC}>50 \times 10^{9} / \mathrm{L}$ were at high risk and $58(69.0 \%)$ patients had $\mathrm{WBC}<50 \mathrm{x}$ $10^{9} / \mathrm{L}$ and were at low risk. $55(65.5 \%)$ patients were treated with BFM protocol and an improvement was seen in overall survival of patients which was $89.9 \%$. After the end of induction $64(73.8 \%)$ patients achieved complete remission, 5 (5.95\%) patients did not achieve remission and there was no record of $13(15.5 \%)$ patients about remission shown in Table-2. FAB classification and immunophenotyping determine the prognosis and treatment of patients having ALL. According to FAB classification, ALL is divided into three subtypes L1, L2, and L3. L1 comprises of small uniform cells and is common in children. L2 comprises small and large cells and is mostly found in adults and L3 comprises varied cells with vacuoles. L3 is called Burkitt's lymphoma /Leukemia. 2 patients had FAB L1, 16 patients had FAB L2 and 2 patients had FAB L3. Immunologically ALL is classified into the categories, $58.3 \%$ of patients had Pre B Cell ALL, (2.38\%) B Cell ALL, (11.9\%) Pre T Cell ALL and (3.57\%) T Cell ALL. Most of the patients had B Cell ALL while T Cell ALL was rare in patients. B Cell ALL have a better prognosis in childhood and those patients are at a low-risk rate while $\mathrm{T}$ Cell ALL patients tend to relapse due to high WBC rate and considered at the high risk (Table-3). BFM-95 protocol was effective in highrisk T-Cell ALL patients as compared to UKALL-XI, MLP-841, and Interfant-99. ${ }^{13}$ Our results were better as compared to results in which high dose of L-asparaginase addition to BFM protocol gave no advantage to ALL patients. ${ }^{12}$ Our findings showed that even though T-Cell ALL are considered high-risk patients, timely treatment with BFM76/79 protocol was effective. BFM 76/79 treatment protocol was offered to 55 patients while 19 patients were treated with different other protocols as shown in Table-3. In terms of percentage $66 \%$ patients were treated with BFM76/79 protocol, 19 patients were treated with different other protocols while $12 \%$ data was missing for ALL patients (Fig-2).

Previous studies exhibited that risk adapted therapies are beneficial for good outcomes of patients in ALL. ${ }^{14,15}$ In the present study, we also attained positive results with the usage of Risk adapted therapy for ALL patients. One patient who was initially treated with BFM 76/79 relapsed in 2nd year of maintenance and then treated with FLAG-TDA had completes remission. Another patient who was initially treated with BFM 76/79 protocol had $44 \%$ blast cells after consolidation, so was treated with HD-MTX (high dose of Methotrexate) and then with FLAG-TDA resulted 
in remission. Nine patients died out of which the death of one patient was due to relapse after the consolidation phase.

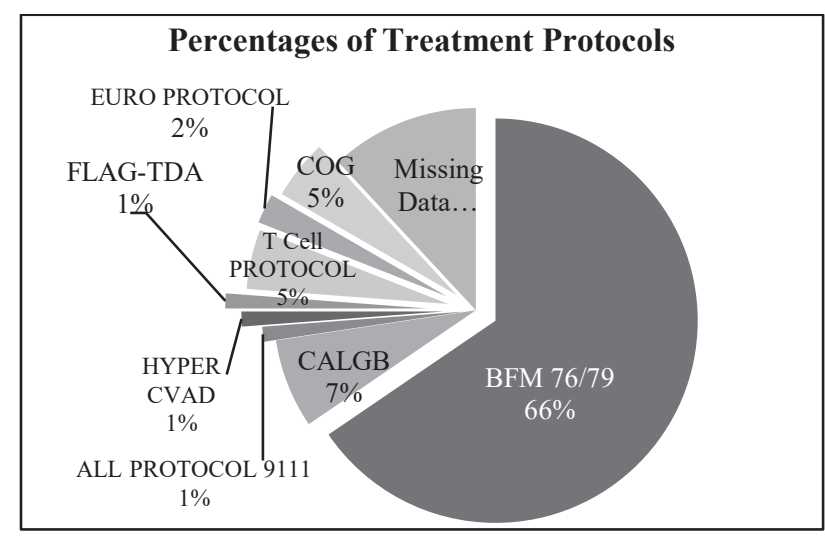

Fig-2: \%age of patients treated with protocols

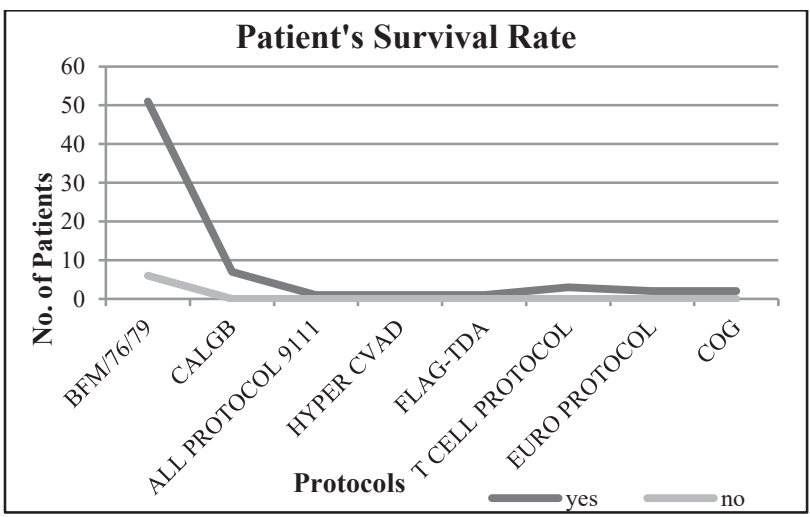

Fig-3: Survival of patients according to a treatment protocol

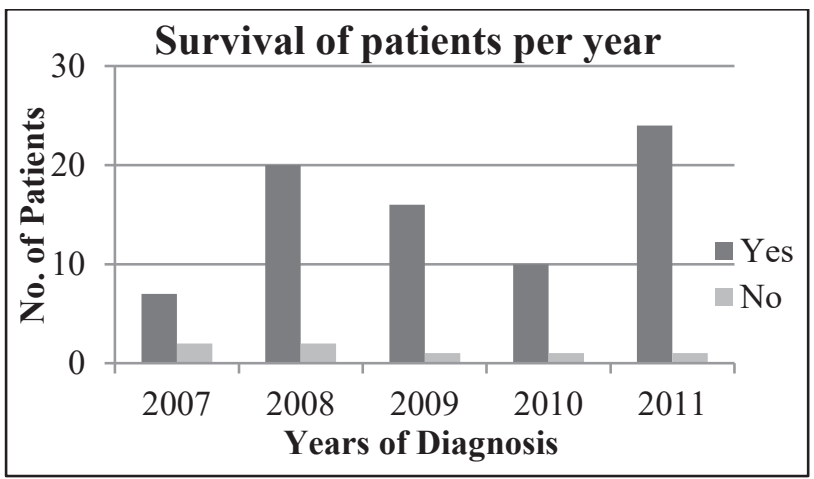

Fig-4: Survival outcomes of ALL patients

. Out of 84 ALL patients only 3 patients had strong leukemic history, so strong leukemic history was not considered as a high risk for leukemia patients. 51 $(65.5 \%)$ patients survived treatment with BFM $76 / 79$ protocol greater than outcomes of other treated protocols. The better outcome obtained was due to greater number of low-risk patients (Fig-3). Overall in our study the relative survival rate per year from 2007 to 2011 overcame the relapse rate and the death rate by treating the patients with risk adapted therapy. The survival rate was higher than the 2007 survival rate but the death rate was almost equal suggests that after treating the patients with risk adapted therapies and controlling the Relapse rate, the survival of patients was increased in 2011 and the death rate was reduced (Fig-4). Our findings concurred with data from other studies wherein the Risk adapted therapies are beneficial for good outcomes of patients in ALL. ${ }^{11,16,17}$ Therefore choice of a protocol has a great impact on the outcome of patients.

Treatment of high-risk patients with suitable or risk directed therapies could result in better outcomes for ALL patients.

\section{CONCLUSION}

The response rate of ALL patients to different chemotherapy protocols was examined. The outcome of), BFM 76/79 protocol was higher than other protocols and possibly when risk adapted for male gender, $\mathrm{WBC}>50 \times 10^{9} / \mathrm{L}$ and age $>30$ years.

\section{Limitations:}

Clearer results could have been obtained from larger data set of patients treated with risk adapted therapies.

\section{Recommendations:}

We can further work on the survival outcomes of different protocols on AML patients.

\section{Acknowledgment:}

We are thankful to Prof. Nadia Sajid, Head of Haematology Department at INMOL, Lahore, and Dr. Abdul Rauf, Associate Professor / Chairperson, Department of Community Medicine, Sargodha Medical College, Sargodha.

\section{REFERENCES}

1. Usman M, Burney I, Nasim A, Adil SN, Salam A, Siddiqui T, Khurshid M. Outcome of Adult Acute Lymphoblastic Leukemia: a Single Center Experience. JPMA 2003: 53(9): 384-388.

2. Idris M, Farid J, Sarwar J, Ahmed S, Wiqar MA, Badsha S. Response rate of Pakistani children with Acute Lymphoblastic Leukemia to Medical Research Council Acute Lymphoblastic Leukemia 97 Chemotherapy Protocol. JAMC 2010; 22(3).

3. BBC News Health: 2013 April 8; Available from: http://www.bbc.co.uk/news/health-2062616

4. DeAngelo DJ. The Treatment of Adolescents and Young Adults with Acute Lymphoblastic Leukemia. Hematology Am SocHematolEduc Program. 2005:123-30. 
5. Tzortzatou-Stathopoulou F, Papadopoulou AL, Moschovi M, Botsonis A, Tsangaris GT. Low relapse rate in children with acute lymphoblastic Leukemia after risk-directed therapy. J PediatrHematolOncol. 2001: 23(9):591-7.

6. BBC News Health: 2010 December 4; Available from:http://www.bbc.co.uk/news/health-1909680.

7. Nwannadi I, Alao O, Bazuaye G, Nwagu M, Borke M. Clinical and Laboratory Characteristics of Patients with Leukaemia in South-South Nigeria. The Internet Journal of Oncology 2011: 7 (2).

8. Wheeler K, Chessells JM, Bailey CC, Richards SM. Treatment related deaths during induction and in first remission in acute lymphoblastic leukaemia: MRC UKALL X. Archives of Disease in Childhood 1996; 74:101-107.

9. Fryer FRCP(C), FACHARTZ SF, Paltiel MS, Khattab MD, Abbas MRCPCH, Yousef MS. Acute Lymphoblastic Leukemia in Children; A Fifteen Year Experience at the Princess Nourah Oncology Center, Jeddah, Saudi Arabia.AustralAsian Journal of Cancer 2006; 5(4):199-204.

10.Abdul-Hamid G. Classification of Acute Leukemia. Acute Leukemia - The Scientist's Perspective and Challenge 2011, Prof. Mariastefania Antica (Ed.), ISBN: 978-953-307553-2.

11.Asim M, Zaidi A, Ghafoor T, Qureshi Y. Death analysis of childhood Acute Lymphoblastic Leukaemia; experience at Shaukat Khanum Memorial Cancer Hospital and Research Centre, Pakistan JPMA 2011: 61(7):666-70.

12.Rizzari C, Valsecchi $\mathrm{MG}$, Aricò $\mathrm{M}$, Conter V, Testi A, Barisone E, Casale F, Lo Nigro L, Rondelli R, Basso G, Santoro N, Masera G. Effect of protracted high-dose L-sparaginase given as a second exposure in a BerlinFrankfurt-Münster-based treatment: results of the randomized 9102 intermediate-risk childhood acute lymphoblastic Leukemia studya report from the Associazione Italiana Ematologia Oncologia Pediatrica. Journal of Clinical Oncology 2001: 19(5):1297-303.

13. Ramzan M, Yadav SP. Does Choice of Treatment Protocol have Impact on outcome in T-cell Lymphoblastic Leukemia. Indian Pediatrics. 2012; 49(248).

14.J Cools J. Improvements in the survival of children and adolescents with acute lymphoblastic Leukemia. Haematologica.2012:97(5):635

15.Pulte D, Gondos A, Brenner. H. Improvement in survival in younger patients with acute lymphoblastic Leukemia from the 1980s to the early 21 st century. Clinical trials and
Observations; Blood 2009: 113(7):1408-1411.

16.Patient information Acute Lymphoblastic Leukemia (ALL): Children, Teenagers and young Adults (to 25 years). UKALL 2011 Trial 2012.

17.Din Mohamed AG, Szabó M, Mark MVD, Visser O, Sonneveld P, Cornelissen JJ, JongenLavrencic M, Rijneveld AW. Improved survival in adult patients with acute lymphoblastic Leukemia in the Netherlands: a populationbased study on treatment, trial participation and survival. Leukemia 2015: 30(2)

\section{The Authors:}

Khansa Saleem,

MS Scholar,

Department of CS \& IT,

University of Sargodha, Sargodha.

Dr. Muhammad Ilyas,

Assistant Professor,

Department of CS \& IT,

University of Sargodha, Sargodha.

Mr. Saad Razzaq,

Assistant Professor,

Department of CS \& IT,

University of Sargodha, Sargodha.

Mr. Fahad Maqbool,

Assistant Professor,

Department of CS \& IT,

University of Sargodha, Sargodha.

Dr. Qaisar Abbas,

Assistant Professor,

Department of CS \& IT,

University of Sargodha, Sargodha.

Dr. Aneem Abdullah,

Postgraduate Resident,

Department of Nephrology,

Shaikh Zayed Medical Complex, Lahore.

Abu Bakar Shahid

Director \& Chief Consultant,

INMOL Hospital, Lahore.

\section{Corresponding Author:}

Dr. Muhammad Ilyas,

Assistant Professor,

Department of CS \& IT,

University of Sargodha, Sargodha

Email: muhammad.ilyas@uos.edu.pk 